\title{
Extruded scintillator for the Calorimetry applications
}

\author{
A. Dyshkant, V. Rykalin \\ Northern Illinois Center for Accelerator and Detector Development (NICADD), \\ Northern Illinois University, DeKalb, IL 60115, USA
}

\author{
A. Pla-Dalmau \\ Fermi Nationa Acceleratorl Laboratory, \\ Batavia, IL 60510, USA \\ D. Beznosko \\ State University of New York at Stony Brook \\ Stony Brook, NY 11790, USA
}

\begin{abstract}
An extrusion line has been installed and successfully operated at FNAL (Fermi National Accelerator Laboratory) in collaboration with NICADD (Northern Illinois Center for Accelerator and Detector Development). This new Facility will serve to further develop and improve extruded plastic scintillator. Recently progress has been made in producing co-extruded plastic scintillator, thus increasing the potential HEP applications of this Facility. The current R\&D work with extruded and co-extruded plastic scintillator for a potential ALICE upgrade, the ILC calorimetry program and the MINERvA experiment show the attractiveness of the chosen strategy for future experiments and calorimetry. We extensively discuss extruded and co-extruded plastic scintillator in calorimetry in synergy with new Solid State Photomultipliers. The characteristics of extruded and co-extruded plastic scintillator will be presented here as well as results with non-traditional photo read-out.
\end{abstract}

Keywords: Extruded scintillator, co-extruded plastic scintillator, calorimeter, Solid State Photomultiplier

PACS: $01.10 . \mathrm{Fv}$ 


\section{INTRODUCTION}

The reason to choose extruded plastic scintillator among the different types of plastic scintillators for Calorimetry applications is based on the attractiveness of using a simple, inexpensive and robust extrusion procedure [1]. Furthermore, in some cases there is no alternative to the extrusion because of geometry requirements. For instance, if you need to build a detector with long strips of different shapes and plastic scintillator is your choice, you have to utilize an extrusion method. The emerging MINERVA experiment [2] intends to use a triangle-shaped extruded scintillator as an active target and calorimeter active media. The triangular shape of the strips and their length (approximately $4 \mathrm{~m}$ long) make the extrusion process an ideal option. In general, the scintillator choice for a given project is based on the compromise between light output, readout system and cost. Cast plastic scintillator may cost between \$40-60 per $\mathrm{kg}$. In contrast, extruded scintillator that has significantly lower price may be used if a wavelength shifting (WLS) fiber readout system is used. With this approach, the MINOS (Main Injector Neutrino Oscillation Search) experiment was able to build two detectors that required almost 300 tons of finished extruded plastic scintillator [3]. The overall cost of the extruded plastic scintillator for MINOS was about $\$ 10$ per $\mathrm{kg}$. The ALICE ECAL upgrade at the LHC needs 15 tons of scintillator. Preliminary R\&D studies [4] have shown the advantages of using extruded plastic scintillator. In the frame of the ILC R\&D program, NICADD proposed using extruded scintillating strips as the active medium for the tail-catcher and muon tracker (TCMT) [4]. NICADD also developed a readout system based on recent development with Solid State Photomultipliers (SSPM) [5]. Coupling with this semiconductor sensor comes in a natural way due to the co-extruded hole and small size of the photo-transducer [6].

In this paper, the basic concept of the extrusion will be illustrated and recent results from measurements testing the response to cosmic muons for different configurations of extruded and co-extruded scintillators will be provided. We hope that the experience accumulated will be useful for different applications in calorimetry and beyond.

\section{EXTRUSION SETUP}

The FNAL-NICADD extrusion line has been operating since April 2003. In 2005 it was upgraded with co-extrusion equipment that allows the placement of a $\mathrm{TiO}_{2}$ white reflective coating on the scintillator strip. The central and vital part of the extrusion line is shown on Fig. 1. From right to left, one sees the 40-mm twin-screw extruder with a die at the end, the co-extruder at 45 degrees above the extruder, and the vacuum cooling tank.

The coating that is a blend of polystyrene and $\mathrm{TiO}_{2}$ is being injected into the die by the co-extruder. From that die, the melt is directed to the sizing tooling located in the vacuum cooling tank. The polystyrene pellets that are the base for the extruded scintillator are a commercial grade, general-purpose polystyrene. The dopants currently in use are $1 \%$ PPO and $0.03 \%$ POPOP, available in a pre-mixed form. This 
is a blue-emitting scintillator with an absorption cut-off at approximately $400 \mathrm{~nm}$ and an emission maximum at $420 \mathrm{~nm}$ [7].

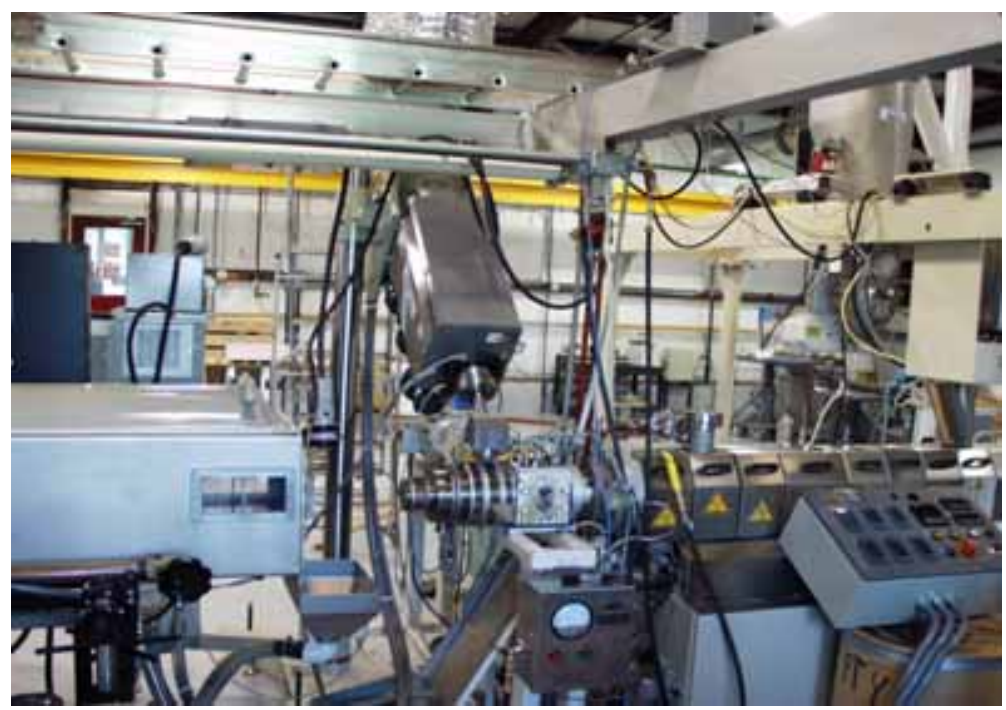

FIGURE 1. Main part of the extrusion setup. From right to left: the 40-mm twin screw extruder with a die at the end, the co-extruder at 45 degrees above the extruder, and the vacuum cooling tank.

\section{EXTRUSION SHAPES WE PRODUCE}

The existing dies produce scintillator strips of different shapes, some with holes for a wavelength shifting (WLS) fiber and some without holes for routing the top surface as needed [7]. The co-extruded hole or groove enables the insertion of the WLS fiber and saves additional expenses. Fig. 2 displays the scintillator profiles with co-extruded holes and white $\mathrm{TiO}_{2}$ coating and other profiles without the white capstocking. The triangular and rectangular-shaped extruded scintillators were chosen by MINERvA as the basic configuration to build the active target and calorimetric part of the detector, respectively.

The scintillator shape depends on the needs of the experiment and its physics demands. For the TCMT prototype, the extruded scintillator strips were produced in the small amount needed for the experiment [8]. Fig. 3 shows the measurements performed on the scintillator production (nominal size: $100 \mathrm{~mm}$ wide by $5 \mathrm{~mm}$ thick). The dimensions had an average width of $101.33 \pm 0.17 \mathrm{~mm}$ evaluated over $300 \mathrm{~m}$ with $50 \mathrm{~cm}$ interval, and an average thickness of $4.98 \pm 0.03 \mathrm{~mm}$ evaluated over $300 \mathrm{~m}$ with $20 \mathrm{~cm}$ interval. 


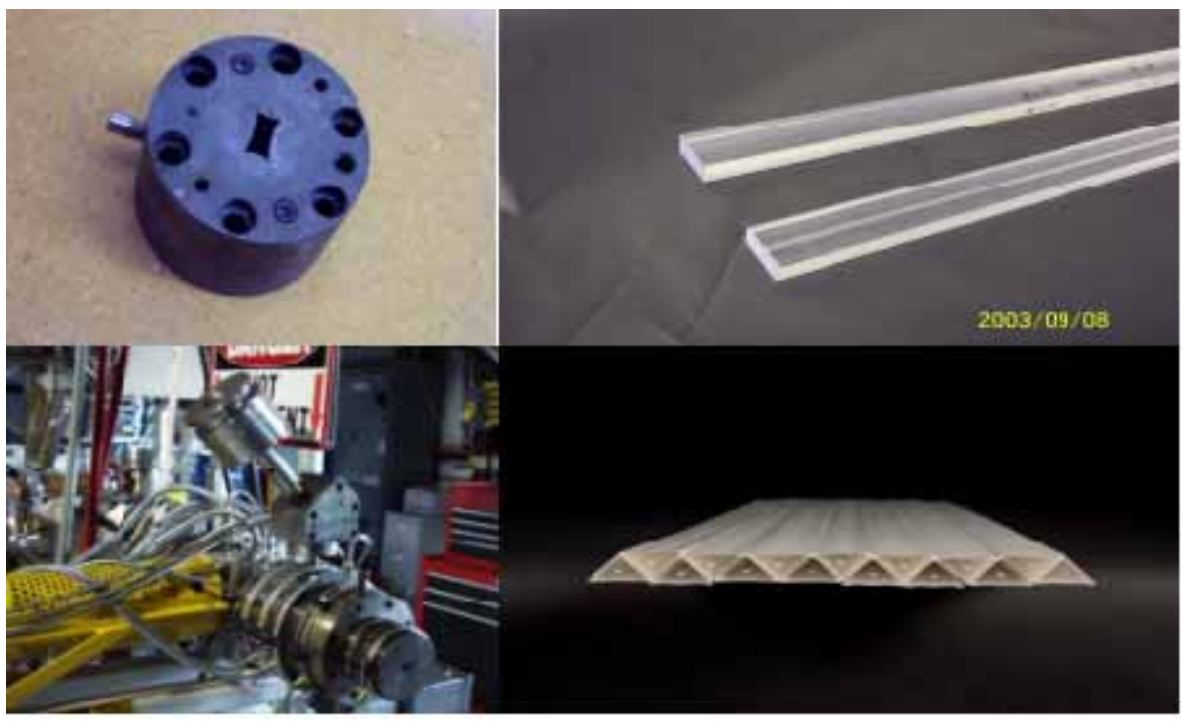

FIGURE 2. Different profiles of scintillator extrusion and dies used to make this particular shape.

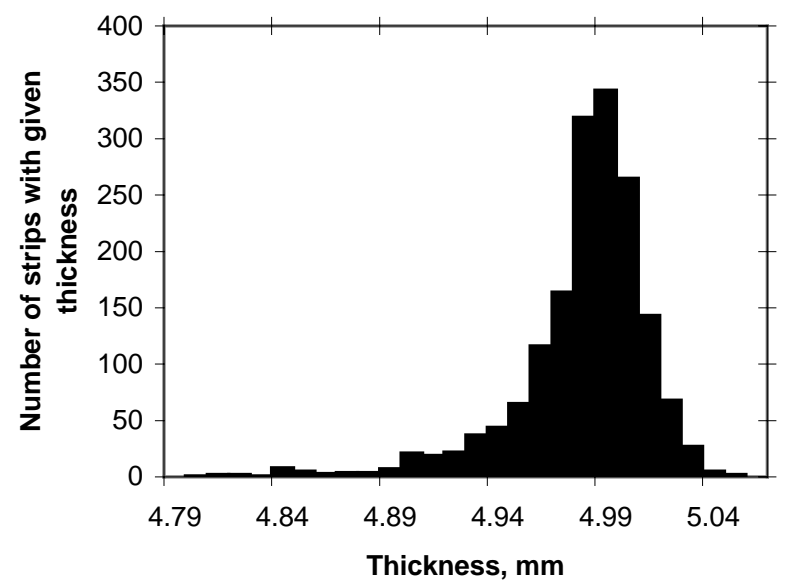

FIGURE 3. Distribution of the strip size with a given thickness.

\section{Experimental results}

\section{Attenuation Length Measurements}

Measurements of the Light Attenuation Length (LAL) [6] show that the value of the long and short components is $42.15 \pm 1.14 \mathrm{~cm}$ and $30.52 \pm 0.74 \mathrm{~cm}$, respectively. The extruded scintillator samples were wrapped in Tyvek since they did not have the co-extruded reflective coating. In order to evaluate the impact of the $\mathrm{TiO}_{2}$ coating, a comparative study was performed using $1 \mathrm{~m}$ long triangular strips. The results are shown on Fig. 4. 


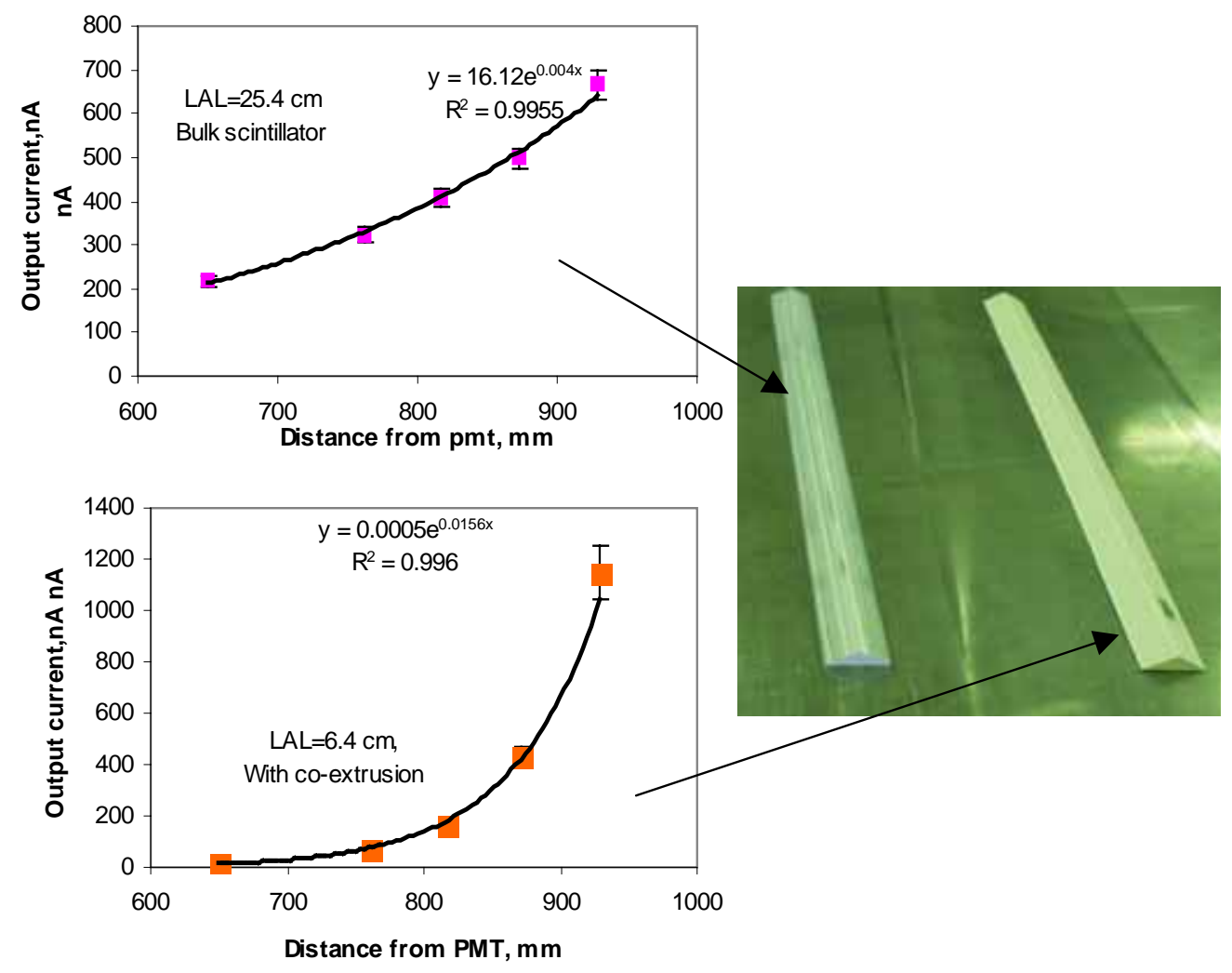

FIGURE 4. Impact of $\mathrm{TiO}_{2}$ co-extrusion on Light Attenuation Length.

It was observed in this set of measurements that the value of the LAL for the extruded scintillator strips (without co-extruded $\mathrm{TiO} 2$ coating) may exceed as much as four times the same value for the $\mathrm{TiO} 2$ co-extruded scintillator strips. The origin of this phenomenon is based on the total internal reflection occurring in the extruded scintillator wrapped in Tyvek that is missing in the $\mathrm{TiO}_{2}$ co-extruded scintillator due to the diffusive character imparted by the coating. It limits a variety of application of this type of scintillator to the use of WLS (Wavelength Shifting) fiber where the LAL is greater than $2 \mathrm{~m}$.

\section{Scintillation Light Yield}

As it was shown in [7], the light yield of our extruded scintillator is of the same value as of Kuraray [9] SCSN-81. The light yield was measured from the extruded tiles using cosmic rays. A peripheral sigma-groove was machined in each tile, where Kuraray Y11 (200ppm) MS WLS fiber with diameter of $0.83 \mathrm{~mm}$ was inserted. The absolute light was estimated using previous measurements of single photoelectron spectra for the Hamamatsu [10] R6094 Photomultiplier Tube (PMT). The tiles produced from two different extruded scintillators averaged to 8 photoelectrons per MIP with 5\% uncertainty on the measurements. 
In order to measure if the size of extruded hole would affect the light yield of the scintillator strips, we have performed measurements on a sample with a variety of hole sizes. Regardless the hole size, we inserted the same $1.2 \mathrm{~mm}$ Kuraray Y11 WLS fiber and measured the resultant light yield while using a ${ }^{137} \mathrm{Cs}$ gamma source. Fig. 5 (top) shows the approximate ratios of the hole size to fiber diameter, with 0.9 being a closest fit in sample used, and 0.3 being very loose. A total of 25 strips were measured. The same figure (bottom) indicates that a better match (0.7-0.9 ratios) does not usually render a higher light yield. The "far Light Yield" and the "near Light Yield" refer to the measurements done in the position farthest from and nearest to the PMT respectively. This measurement illustrates that the hole size does not have a significant impact on the light yield.

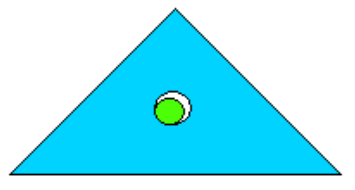

0.9

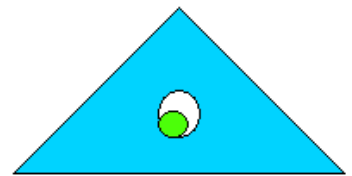

0.5

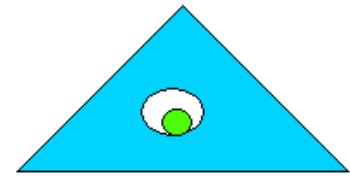

0.3

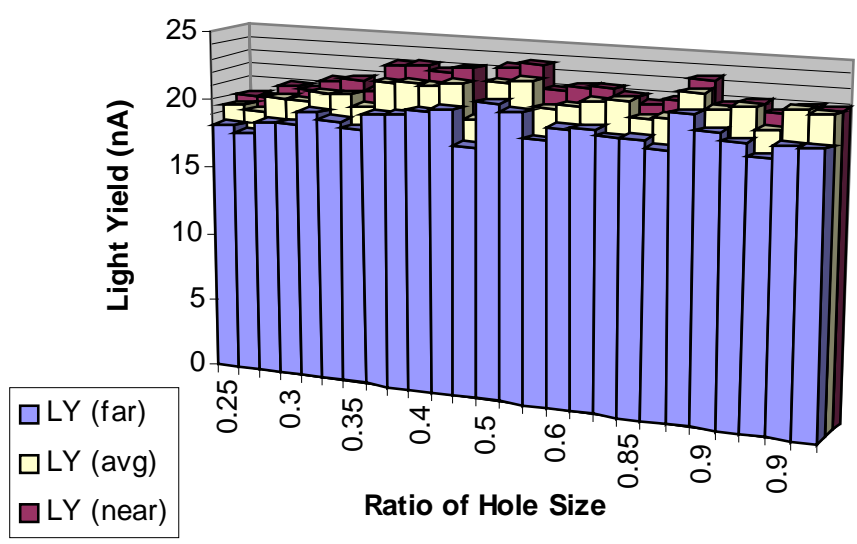

FIGURE 5. Effect on Light Yield due to the Hole Sizes

Several measurements using a Solid State Photomultiplier (SSPM) were also performed. This is a multi-pixel solid-state device with every pixel operating in the Limited Geiger multiplication mode. Avalanche quenching is achieved by means of a resistive layer on the sensor surface. The device has about 1500 pixels per $1 \mathrm{x} 1 \mathrm{~mm}^{2}$ sensor [5]. The quantum efficiency (QE) of the device is approximately $20 \%$ at 500 $\mathrm{nm}$ : a value that is comparable to the $\mathrm{QE}$ of a conventional green-extended PMT.

The dynamic range of the sensor fits many applications where the linearity issue is of importance. Deviation from linearity at the level of 5\% was observed [5] at approximately 2200 photons of input signal and deviation from linearity at the level of $10 \%$ was seen with light intensity of about 3200 photons, as shown in Fig. 6. 


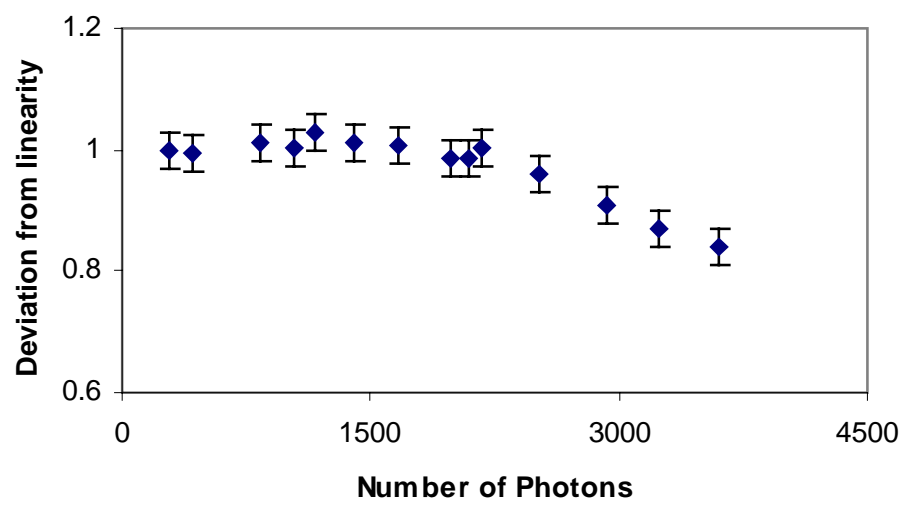

FIGURE 6. Dynamic range of SSPM
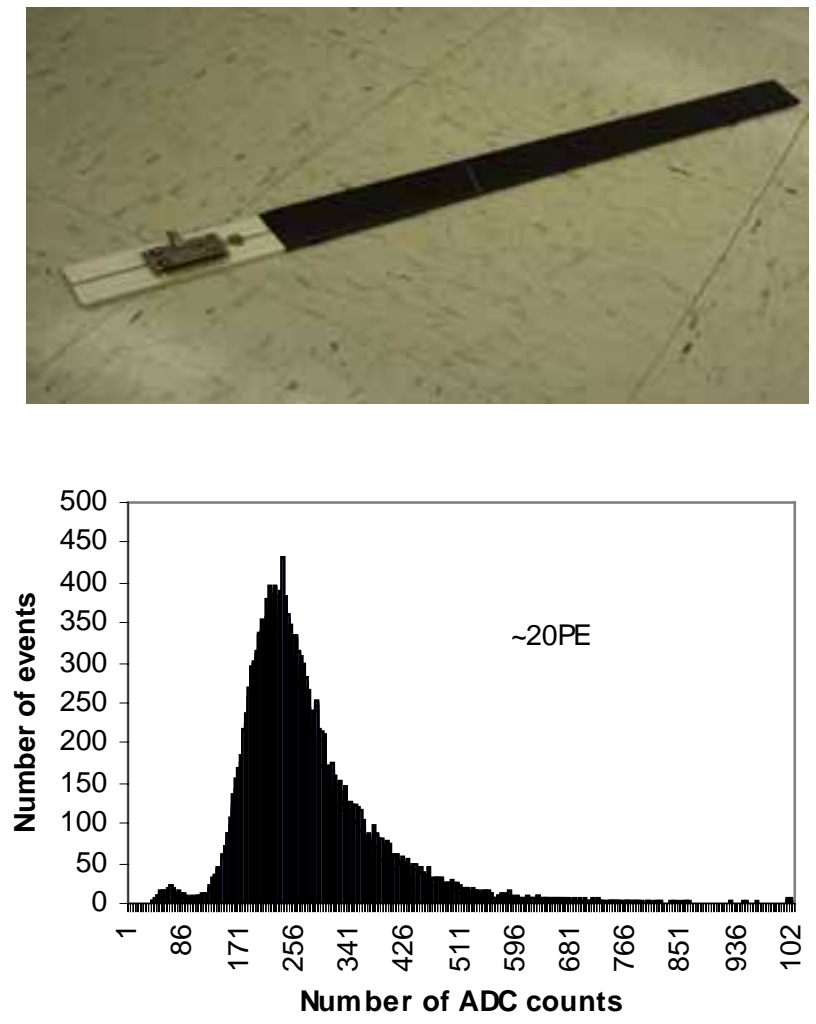

FIGURE 7. Cosmic counter assembly on the top and amplitude spectra from cosmic muons

The coupling of the SSPM and the WLS fiber comes in natural way [6]. Several scintillator counters for a cosmic-ray test stand were built with SSPM photo-readout. For the prototype a MINOS strip was utilized with a WLS fiber in the co-extruded groove. The SSPM sensor and biasing circuit was placed on the same strip for portability (Fig. 7, top). The cosmic-ray signal spectrum is shown on Fig. 7 (bottom). The pedestal is slightly smeared due to the geometry of the trigger. The 
detector has a perfect single photoelectron (PE) distribution that was measured before the amplitude distribution was accumulated for calibration purpose. The estimated number of photelectrons for this spectrum is about 20 PE. Fig. 7 also indicates that the signal is well separated from the pedestal. Since SSPM is insensitive to strong magnetic fields [11], it is an attractive option for detectors where a strong field is an issue.

\section{CONCLUSION}

The FNAL-NICADD Extrusion Line is producing a variety of scintillator shapes and sizes, including co-extruded hole and $\mathrm{TiO}_{2}$ coating. The scintillator bars with co-extruded capstocking can be used in conjunction with WLS fibers in calorimeters that require long and inexpensive strips, like the MINOS and MINERvA experiments. The use of SSPM with the extruded scintillator yields attractive results for their combined use in future experiments.

\section{ACKNOWLEDGMENTS}

The authors would like to thank Chuck Serritella and Elena Baldina for general support and assistance during the R\&D extrusion work. We also would like to thank Daniel Ruggiero for providing the Y11 fibers.

\section{REFERENCES}

1. D. Beznosko, A. Bross, A. Dyshkant, A. Pla-Dalmau, V. Rykalin, "Production Using FNAL-NICADD Extruder", FERMILAB-PUB-05-344, Jul 29, 2005

2. D. Drakoulakos et al. [the MINERvA collaboration], "Proposal to Perform a High-Statistics Neutrino Scattering Experiment Using a Fine-grained Detector in the NuMI Beam". http://www.pas.rochester.edu/minerva/

3. MINOS Press Releases, http://www.fnal.gov/pub/presspass/press_releases/minos_3-30-06.html

4. A. Dyshkant, D. Beznosko, G. Blazey, D. Chakraborty, K. Francis, D. Kubik et al., "Towards a ScintillatorBased Digital Hadron Calorimeter for the Linear Collider Detector", IEEE vol. 51, no. 4, pp.1590-1595, Aug. 2004.

5. D. Beznosko, G. Blazey, D. Chakraborty, A. Dyshkant, K. Francis, D. Kubik et al., "Investigation of a Solid State Photodetector", NIM A 545 (2005) 727-737

6. D. Beznosko, P. Polozov, V. Rykalin, G. Sellberg, "Fiber Positioning and MRS Response ", FERMILAB-TM2247, May 7, 04

7. D. Beznosko, A. Bross, A. Dyshkant, A. Pla-Dalmau V. Rykalin, "Production Using FNAL-NICADD Extruder", FERMILAB-PUB-05-344, Jul 29, 2005

8. A. Dyshkant, E. Baldina, D. Beznosko, G. Blazey, A. Bross, D. Chakraborty et al., "Studies of NICADD Extruded Scintillator Strips", FERMILAB-CONF-05-243, June 20, 2005

9. Kuraray America Inc., 200 Park Ave, NY 10166,USA; 3-1-6, NIHONBASHI, CHUO-KU, TOKYO 103-8254, JAPAN

10. Hamamatsu Corporation, 360 Foothill Road, PO Box 6910, Bridgewater, NJ 08807-0919,USA

11. D. Beznosko, G. Blazey, A. Dyshkant, V. Rykalin, J. Schellpffer, V. Zutshi, "Modular Design for Narrow Scintillating Cells with MRS Photodiodes in Strong Magnetic Field for ILC Detector", accepted to NIM A, Apr 16 2006, NIMA-D-05-00459 